\title{
The immunology of blood: connecting the dots at the neurovascular interface
}

\author{
Katerina Akassoglou \\ Gladstone Institutes and Department of Neurology, Weill Institute of Neurosciences, University of \\ California, San Francisco, 1650 Owens St., San Francisco, CA 94158, USA.
}

In 1883, Eduard von Rindfleisch identified multiple sclerosis (MS) lesions around vessels "engorged with blood", providing the first link between blood leaks and brain inflammation ${ }^{1}$. Since then, blood extravasation has been shown to be a common thread in many devastating brain and peripheral diseases, correlating with disease severity. However, a chicken-and-egg question has posed a long-standing dilemma: does more blood leak into the brain as a consequence of disease, or does the influx of blood proteins enable disease pathogenesis? Here, I focus on my 26-year journey to connect the dots between blood, the inflammatory response, and brain disease. Together with an international team of scientists and collaborators, my work shed light on the causal role of blood leaks in toxic inflammation and tissue damage, and revealed a unique niche at the neurovascular interface for the development of imaging tools and new therapeutics ${ }^{2-4}$.

\section{TNF days}

In 1994, I started my PhD work studying Tumor Necrosis Factor (TNF) in the laboratory of George Kollias at the Hellenic Pasteur Institute in Athens, Greece. The Kollias lab had just discovered that transgenic mice expressing TNF in the synovium spontaneously developed severe inflammation in their joints, indicating a direct involvement of TNF in rheumatoid arthritis (RA) ${ }^{5}$. I was fortunate to participate in the preclinical testing of anti-TNF monoclonal antibodies that were later approved for the treatment of RA ${ }^{6}$. However, as all the arthritis-related $\mathrm{PhD}$ projects were taken, for my thesis I was assigned to resolve the enigmatic phenotype of a paralyzed TNF transgenic mouse line without signs of arthritis. In the Kollias lab, we found that in this mouse model TNF was expressed only in the brain and spinal cord resulting in features reminiscent of MS, such as neuroinflammation, demyelination, and paralysis. The finding that expression of a cytokine in the brain was sufficient to induce demyelination and paralysis without immunizing with myelin antigens was so unexpected at the time that my short oral presentation at the IV International Society

kakassoglou@gladstone.ucsf.edu.

Competing interests

K.A. is the scientific founder and advisor of Therini Bio, Inc. K.A. is an inventor on The Regents of the University of California issued patents US7807645, US8569242, US8877195, and US8980836 covering fibrin antibodies; a co-inventor on The Regents of the University of California and Gladstone Institutes pending patent applications U.S. 15/943,474 and EP 15735033.1 covering fibrin assays of microglia activation; and a co-inventor on Gladstone Institutes issued patent US9669112 covering fibrin in vivo models, US10451611 and pending patent applications U.S. 16/572,365 and EP 15735284.05 covering in vitro fibrin assays. Her interests are managed in accordance with the Gladstone Institutes' conflict of interest policy. 
of Neuroimmunology (ISNI) Congress in Amsterdam in 1994 was featured in the meeting highlights ${ }^{7}$. A year later, our paper reporting spontaneous inflammatory demyelination by TNF expression in the brain was communicated by Rita Levi-Montalcini to the Proceedings of the National Academy of Sciences. Building upon these findings, I generated multiple transgenic lines that revealed that TNF produced by astrocytes, but not neurons was responsible for the neurologic disease ${ }^{8}$. We attempted to genetically rescue the TNF transgenic mice by depleting $\mathrm{T}$ cells, but surprisingly found that $\mathrm{T}$ cell depletion exacerbated features of the disease ${ }^{9}$. This result led us to conclude that innate immunity was responsible for demyelination, and made us question the prevailing hypothesis that $\mathrm{T}$ cell suppression would be therapeutic in progressive disease ${ }^{9}$. But if $\mathrm{T}$ cells were not the mediators of inflammatory demyelination in the TNF transgenic mice, what was triggering the disease?

\section{Finding fibrin in Vienna}

I presented my findings in the TNF transgenic mice to the Demyelination and Remyelination INSERM Conference Philippe Laudat at Aix-Les-Bains in 1995. After a talk in which I faced more questions than I could answer, I realized how essential it would be to master neuropathology in order to understand how innate immune activation induced brain disease. During the meeting social on a boat tour of the Rhône river, I met Hans Lassmann, an expert MS neuropathologist at the Medical University of Vienna. Dr. Lassmann was intrigued by my presentation, and we brainstormed ways to experimentally determine the mechanism of disease in the TNF transgenic mice. In the fall of 1996, with support from the European Molecular Biology Organization, I moved to his laboratory in Vienna to analyze hundreds of brains from the TNF transgenic mice. A key finding from these studies was axonal damage and oligodendrogliopathy induced by innate immune activation, suggesting that TNF transgenic mice could be a model to study $\mathrm{MS}^{10}$. To find out how this pathology started, we analyzed the brains early, before any symptoms occurred. Using electron microscopy, I noticed major changes in blood vessels and perivascular deposits, which Dr. Lassmann identified as massive blood-brain barrier (BBB) disruptions with fibrin deposits. We could detect BBB leaks as early as one week of age, before any other symptom or cell infiltration was present. Had we found the smoking gun? As fibrinogen is a marker for BBB disruption, we looked for fibrinogen immunostaining in the brains of MS patients. I was awed to discover an abundance of fibrin deposits, which often colocalized with microglia and macrophages. Eager to learn more about fibrin, I found a paper that had just been published reporting that fibrin mediates inflammation via the $\mathrm{CD} 11 \mathrm{~b} / \mathrm{CD} 18$ integrin receptor ${ }^{11}$. These observations and results, in combination with our earlier finding that astrocytes-which regulate the $\mathrm{BBB}$ - were the primary source of TNF promoting neuroinflammation ${ }^{8}$, led me to hypothesize that $\mathrm{BBB}$ disruption is an upstream activator of innate immune-mediated demyelination and neurodegeneration.

At the time, fibrin was primarily studied by hematologists for its role in blood coagulation, which made my search for a laboratory in which to study its role in brain inflammation unfruitful. However, in 1997, I met Sidney Strickland at the 17th Blankenese Conference on Neurodegeneration in Hamburg, shortly after his landmark paper on the role of the fibrinolytic enzyme, tissue plasminogen activator (tPA), in excitotoxic neuronal death ${ }^{12}$. I joined his laboratory as a postdoctoral fellow, first at the State University of New York at 
Stony Brook and later at The Rockefeller University, to determine the role of the tPA/fibrin pathway in neuroinflammation. Integrating vascular and BBB research in neuroimmunology was unconventional at the time and many assumed I had changed fields. In Dr. Strickland's laboratory, I first found that tPA- and plasminogen-deficient mice had exacerbated demyelination after sciatic nerve injury, which could be rescued by fibrin depletion ${ }^{13}$. In collaboration with Jay Degen at Cincinnati Children's Hospital, we performed the first genetic validation of the role of fibrinogen in the nervous system ${ }^{14}$. Mice lacking fibrinogen had increased remyelination after nerve injury and decreased neuroinflammation and demyelination in the context of transgenic TNF expression ${ }^{13,15,16}$. These results were in line with protective effects of anti-coagulants in autoimmune MS models ${ }^{17}$. In addition, fibrinogen-deficient mice had increased expression of the $\mathrm{p} 75$ neurotrophin receptor $\left(\mathrm{p} 75^{\mathrm{NTR}}\right.$ ) after nerve injury ${ }^{15}$. In the laboratory of Moses Chao at New York University, I made the serendipitous discovery that tPA expression and fibrinolysis were suppressed by $\mathrm{p} 75^{\mathrm{NTR}}$, identifying an unanticipated role for neurotrophin receptor signaling in fibrin removal from the nervous system ${ }^{18}$. Fibrinogen-deficient mice were later studied in other laboratories and their protection from neuroinflammation and demyelination was confirmed in mouse models of Alzheimer's, pericyte-deficiency, and brain injury ${ }^{19-21}$. But how did fibrin induce these effects on the brain's immune and repair functions?

\section{Zeroing in on innate immunity}

In 2002, in a review article entitled "Nervous system pathology: the fibrin perspective", we first proposed the hypothesis that fibrin interacts with receptors on nervous system cells ${ }^{22}$. In my laboratory at the University of California San Diego and Gladstone Institutes, we discovered the cell targets for fibrinogen and fibrin in the brain and identified a causal role for BBB disruption in glial activation and neuronal dysfunction ${ }^{23-26}$. We discovered that culturing microglia on fibrin-coated plates induced dramatic activation ${ }^{26}$. Fibrinogen binds the CD11b/CD18 receptor via the fibrin $\gamma_{377-397}$ peptide $^{27}$ and genetic mutation of that peptide ( $F g g$ r390-396A mice) results in fully coagulable fibrinogen which does not bind the $\mathrm{CD} 11 \mathrm{~b} / \mathrm{CD} 18^{28}$. This allowed us to investigate the role of fibrin-induced inflammation in the brain without disrupting hemostasis. We showed that Fgg $7390-396 \mathrm{~A}$ mice had reduced neurological signs, inflammation, demyelination, and perivascular microglia clusters in EAE - a mouse model of $\mathrm{MS}^{26,29}$. By in vivo two-photon imaging, we showed that fibrin deposition and coagulation activity occurred and correlated with microglial activation ${ }^{29-31}$. Fibrin signaling via CD11b/CD18 was sufficient to induce demyelination and peripheral cell recruitment ${ }^{32}$, as well as oxidative stress via activation of NADPH oxidase ${ }^{4}$; a mechanism that also contributes to microglia-mediated synapse loss in Alzheimer's disease models ${ }^{33}$. Blood clotting and oxidative stress genes are mechanistically coupled in neuroinflammation, suggesting a positive feedback loop between fibrin, inflammation, coagulation and oxidative injury ${ }^{35}$. These results established that interaction of fibrin with an inflammatory receptor was necessary and sufficient to promote neuroinflammation (Fig. 1). Could we develop an approach to selectively target the inflammatory functions of fibrin without affecting its beneficial effects in hemostasis? 


\section{The making of an antibody}

During my postdoc at The Rockefeller University, we occasionally had joint lab meetings with Barry Coller, the inventor of ReoPro ${ }^{\mathrm{TM}}$ (abciximab), a monoclonal antibody used as a thrombolytic that potently inhibits fibrinogen binding to $\alpha_{\mathrm{IIb}} \beta_{3}$ platelet integrin ${ }^{34}$. Thinking back on his work, I wondered: if an antibody can specifically inhibit the binding of fibrin to platelets and reduce thrombus formation, could we develop an antibody to specifically inhibit binding of fibrin to immune cells and stop inflammation without interfering with hemostasis? As described above, the fibrinogen $\gamma$ chain contains the $\gamma_{377-395}$ peptide that mediates interaction with $\mathrm{CD} 11 \mathrm{~b} / \mathrm{CD} 18^{27}$, and this is adjacent to but distinct from the motif that mediates platelet engagement. The $\gamma_{377-395}$ peptide is considered cryptic in soluble fibrinogen, and binding to CD11b occurs only after the conversion of fibrinogen to insoluble fibrin ${ }^{27}$. I hypothesized that a monoclonal antibody against the $\gamma_{377-395}$ epitope would selectively inhibit the interaction between fibrin and CD11b without affecting coagulation. This hypothesis was supported by our finding that mutating the $\gamma_{377-395}$ epitope (in Fgg $\gamma 390-396 A$ mice) or administering $\gamma_{377-395}$ peptide reduced neuroinflammation without interfering with in vivo clotting time ${ }^{26}$. In my laboratory, we generated antibodies against the $\gamma_{377-395}$ peptide and the lead clone was 5B8, a highly selective monoclonal antibody that selectively bound fibrin and blocked fibrin-induced, CD11b-mediated activation of innate immunity without affecting fibrin polymerization ${ }^{4}$. As drug discovery requires broad expertise, I put together a multi-disciplinary team of thirty-four scientists in three academic institutions and pharma to fully characterize this fibrin-targeting immunotherapy ${ }^{4}$. Jae Kyu Ryu and Anke Meyer-Franke led the development of biochemical and cell assays to study fibrin in vitro that enabled the screening of fibrin-targeting antibody clones and their prioritization for in vivo studies ${ }^{4}$. In accordance with the genetic evidence in $\mathrm{Fgg}^{2} 390-396 \mathrm{~A}$ mice $^{26,33}$, we found that the fibrin-targeting immunotherapy protected mouse models of EAE and Alzheimer's disease from neuroinflammation and neurodegeneration ${ }^{4}$ (Fig. 1).

As neurovascular interactions are complex and multifactorial, one might ask whether targeting a single blood protein will be enough to protect from neuroinflammation. A similar question was raised in the early nineties regarding anti-TNF therapy, as noted by Jan Vilcek, the inventor of Remicade ${ }^{\mathrm{TM}}$ (infliximab): "To most scientists and physicians it seemed inconceivable that blocking a single cytokine could be beneficial for $R A$ patients, when it was known that multiple cytokines are involved in the inflammatory process" ${ }^{\prime}$. Despite this complexity, targeting individual proteins within the cytokine or angiogenic networks, such as TNF or VEGF, has indeed succeeded in the clinic. As fibrin is a global activator of innate immunity at sites of vascular damage ${ }^{3}$, these approaches could have therapeutic benefits in brain and peripheral diseases. Therefore, fibrin-targeting immunotherapy, a novel approach to selectively suppress pathogenic innate immunity at sites of vascular damage, may have therapeutic benefits in clinical applications.

\section{What the future holds}

The interface of the brain, immune, and vascular systems represents a new frontier of scientific exploration, with the potential to change the way we think about fundamental mechanisms of neurological diseases and discovery of novel therapies" . "Connecting the 
dots" will continue to depend on an unwavering focus on the discovery of new mechanisms, pursuing the integration of new technologies, and promoting collaborations within a community of experts in neuroscience, immunology, vascular biology, drug discovery, and clinical development, all the while welcoming serendipity. Developing safe and efficacious drugs against well-defined necessary and sufficient targets, such as fibrin, could expand the toolbox of therapies to target neurovascular pathology. Implementing functional multiomic approaches will further reveal new mechanisms and therapeutic targets at the neurovascular interface ${ }^{35}$. As the neurovascular interface is a unique niche for the development of imaging tools and new therapies, drug discovery selectively targeting pathogenic functions of blood proteins can change the diagnosis and treatment of neurodegenerative, autoimmune and inflammatory diseases.

\section{Acknowledgements}

I am especially grateful to all my current and former laboratory members for their creativity, grit, and exceptional contributions. I thank my mentors, collaborators, and colleagues, including those that I was unable to mention here. Thanks to Hans Lassmann, Sidney Strickland, Jay L. Degen, George Kollias, Moses V. Chao, Mark A. Petersen, and Kathryn Claiborn for critical reading of this manuscript. K.A. is currently supported by NIH/NINDS R35 NS097976, NIH/NIA RF1 AG064926, DoD MS160082, the Dagmar Dolby Family Fund, gift from Edward and Pearl Fein, and the Simon Family Trust. Lastly, I am grateful to the University of California, San Diego, Gladstone Institutes, and University of California, San Francisco for promoting a culture of discovery and innovation.

Katerina Akassoglou recounts how her work on the mechanisms and functions of blood leaks in the brain led to the discovery of fibrin-targeting immunotherapy

\section{References}

1. Rindficisch E. Histologisches detail zu der grauen degeneration von gehirn und ruckenmark. Archives of Pathological Anatomy and Physiology 26, 474-483 (1863).

2. Petersen MA, Ryu JK \& Akassoglou K. Fibrinogen in neurological diseases: mechanisms, imaging and therapeutics. Nat. Rev. Neurosci 19, 283-301 (2018). [PubMed: 29618808]

3. Davalos D \& Akassoglou K. Fibrinogen as a key regulator of inflammation in disease. Semin. Immunopathol 34, 43-62 (2012). [PubMed: 22037947]

4. Ryu JK. et al. Fibrin-targeting immunotherapy protects against neuroinflammation and neurodegeneration. Nat. Immunol 19, 1212-1223 (2018). [PubMed: 30323343]

5. Keffer J. et al. Transgenic mice expressing human tumour necrosis factor: a predictive genetic model of arthritis. EMBO J. 10, 4025-4031 (1991). [PubMed: 1721867]

6. Vilcek J. Love and science: a memoir (Seven Stories Press, New York, 2016).

7. Cross AH, Vincent A \& Racke MK IVth Congress of the International Society of Neuroimmunology Amsterdam, The Netherlands, 23-27 October 1994. J. Neuroimmunol 58, $117-$ 120 (1995). [PubMed: 7730447]

8. Akassoglou K, Probert L, Kontogeorgos G \& Kollias G. Astrocyte-specific but not neuron-specific transmembrane TNF triggers inflammation and degeneration in the central nervous system of transgenic mice. J. Immunol 158, 438-445 (1997). [PubMed: 8977220]

9. Kassiotis G. et al. A tumor necrosis factor-induced model of human primary demyelinating diseases develops in immunodeficient mice. Eur. J. Immunol 29, 912-917 (1999). [PubMed: 10092095]

10. Akassoglou K. et al. Oligodendrocyte apoptosis and primary demyelination induced by local TNF/ p55TNF receptor signaling in the central nervous system of transgenic mice: models for multiple sclerosis with primary oligodendrogliopathy. Am. J. Pathol 153, 801-813 (1998). [PubMed: 9736029]

11. Tang L, Ugarova TP, Plow EF \& Eaton JW Molecular determinants of acute inflammatory responses to biomaterials. J. Clin. Invest 97, 1329-1334. (1996). [PubMed: 8636446] 
12. Tsirka SE, Gualandris A, Amaral DG \& Strickland S. Excitotoxin-induced neuronal degeneration and seizure are mediated by tissue plasminogen activator. Nature 377, 340-344. (1995). [PubMed: 7566088]

13. Akassoglou K, Kombrinck KW, Degen JL \& Strickland S. Tissue plasminogen activator-mediated fibrinolysis protects against axonal degeneration and demyelination after sciatic nerve injury. J. Cell Biol 149, 1157-1166 (2000). [PubMed: 10831618]

14. Bugge TH et al. Loss of fibrinogen rescues mice from the pleiotropic effects of plasminogen deficiency. Cell 87, 709-719 (1996). [PubMed: 8929539]

15. Akassoglou K, Yu W-M, Akpinar P \& Strickland S. Fibrin inhibits peripheral nerve regeneration by arresting Schwann cell differentiation. Neuron 33, 861-875 (2002). [PubMed: 11906694]

16. Akassoglou K. et al. Fibrin depletion decreases inflammation and delays the onset of demyelination in a tumor necrosis factor transgenic mouse model for multiple sclerosis. Proc. Natl. Acad. Sci. U S A 101, 6698-6703 (2004). [PubMed: 15096619]

17. Paterson PY Experimental allergic encephalomyelitis: role of fibrin deposition in immunopathogenesis of inflammation in rats. Fed. Proc 35, 2428-2434. (1976). [PubMed: 61895]

18. Sachs BD et al. p75 neurotrophin receptor regulates tissue fibrosis through inhibition of plasminogen activation via a PDE4/cAMP/PKA pathway. J. Cell Biol 177, 1119-1132 (2007). [PubMed: 17576803]

19. Cortes-Canteli M. et al. Fibrinogen and beta-amyloid association alters thrombosis and fibrinolysis: a possible contributing factor to Alzheimer's disease. Neuron 66, 695-709 (2010). [PubMed: 20547128]

20. Montagne A. et al. Pericyte degeneration causes white matter dysfunction in the mouse central nervous system. Nat. Med 24, 326-337 (2018). [PubMed: 29400711]

21. Pous L. et al. Fibrinogen induces neural stem cell differentiation into astrocytes in the subventricular zone via BMP signaling. Nat. Commun 11, 630 (2020). [PubMed: 32005867]

22. Akassoglou K \& Strickland S Nervous system pathologies: The fibrin perspective. Biol. Chem 383, 37-45 (2002). [PubMed: 11928820]

23. Schachtrup C. et al. Fibrinogen inhibits neurite outgrowth via beta3 integrin-mediated phosphorylation of the EGF receptor. Proc. Natl. Acad. Sci. U S A 104, 11814-11819 (2007). [PubMed: 17606926]

24. Petersen MA et al. Fibrinogen Activates BMP Signaling in Oligodendrocyte Progenitor Cells and Inhibits Remyelination after Vascular Damage. Neuron 96, 1003-1012 e1007 (2017).

25. Schachtrup C. et al. Fibrinogen triggers astrocyte scar formation by promoting the availability of active TGF-beta after vascular damage. J. Neurosci 30, 5843-5854 (2010). [PubMed: 20427645]

26. Adams RA et al. The fibrin-derived gamma377-395 peptide inhibits microglia activation and suppresses relapsing paralysis in central nervous system autoimmune disease. J. Exp. Med 204, 571-582 (2007). [PubMed: 17339406]

27. Ugarova TP et al. Sequence gamma 377-395(P2), but not gamma 190-202(P1), is the binding site for the alpha MI-domain of integrin alpha $\mathrm{M}$ beta 2 in the gamma C-domain of fibrinogen. Biochemistry 42, 9365-9373 (2003). [PubMed: 12899623]

28. Flick MJ et al. Leukocyte engagement of fibrin(ogen) via the integrin receptor alphaMbeta2/Mac-1 is critical for host inflammatory response in vivo. J. Clin. Invest 113, 1596-1606 (2004). [PubMed: 15173886]

29. Davalos D. et al. Fibrinogen-induced perivascular microglial clustering is required for the development of axonal damage in neuroinflammation. Nat. Commun 3, 1227 (2012). [PubMed: 23187627]

30. Davalos D. et al. Early detection of thrombin activity in neuroinflammatory disease. Ann. Neurol 75, 303-308 (2014). [PubMed: 24740641]

31. Lee NJ et al. Spatiotemporal distribution of fibrinogen in marmoset and human inflammatory demyelination. Brain 141, 1637-1649 (2018). [PubMed: 29688408]

32. Ryu JK et al. Blood coagulation protein fibrinogen promotes autoimmunity and demyelination via chemokine release and antigen presentation. Nat. Commun 6, 8164 (2015). [PubMed: 26353940]

33. Merlini M. et al. Fibrinogen induces microglia-mediated spine elimination and cognitive impairment in Alzheimer's Disease. Neuron 101, 1099-1108 (2019). [PubMed: 30737131] 
34. Coller BS, Anderson KM \& Weisman HF The anti-GPIIb-IIIa agents: fundamental and clinical aspects. Haemostasis 26 Suppl 4, 285-293 (1996). [PubMed: 8979134]

35. Mendiola AS, et al. Transcriptional profiling and therapeutic targeting of oxidative stress in neuroinflammation. Nat. Immunol (2020) in press. 


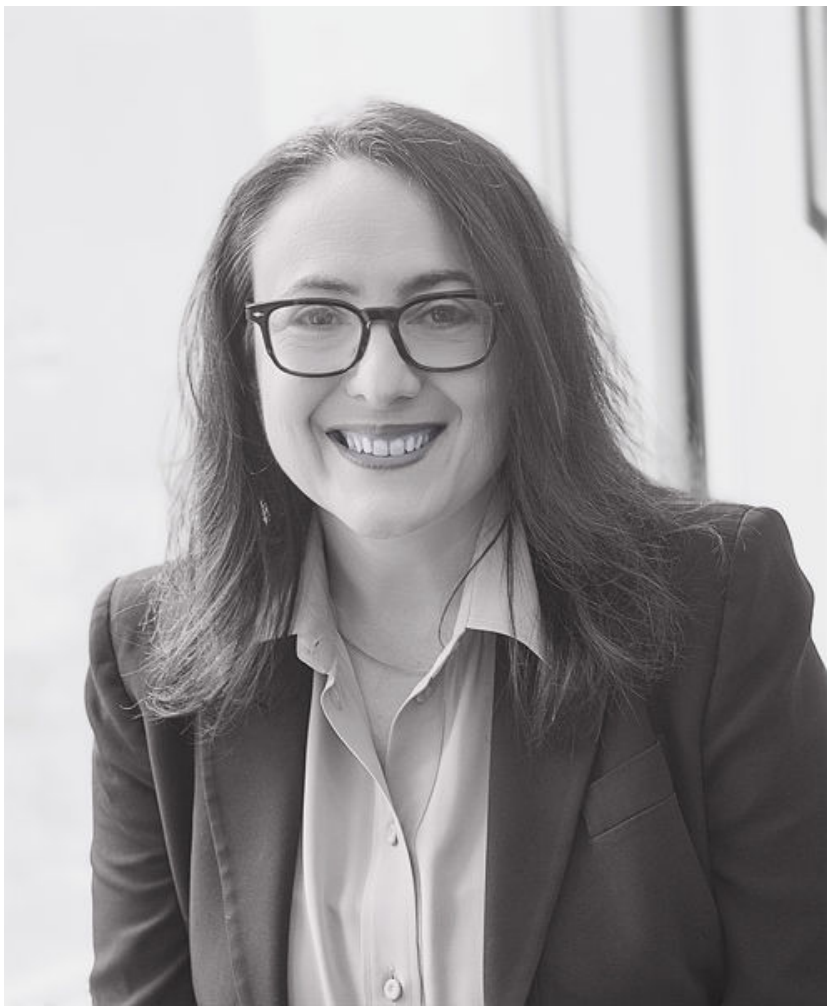

Photo:

Katerina Akassoglou, $\mathrm{PhD}$ in her laboratory at Gladstone Institutes, University of California San Francisco in 2019. 

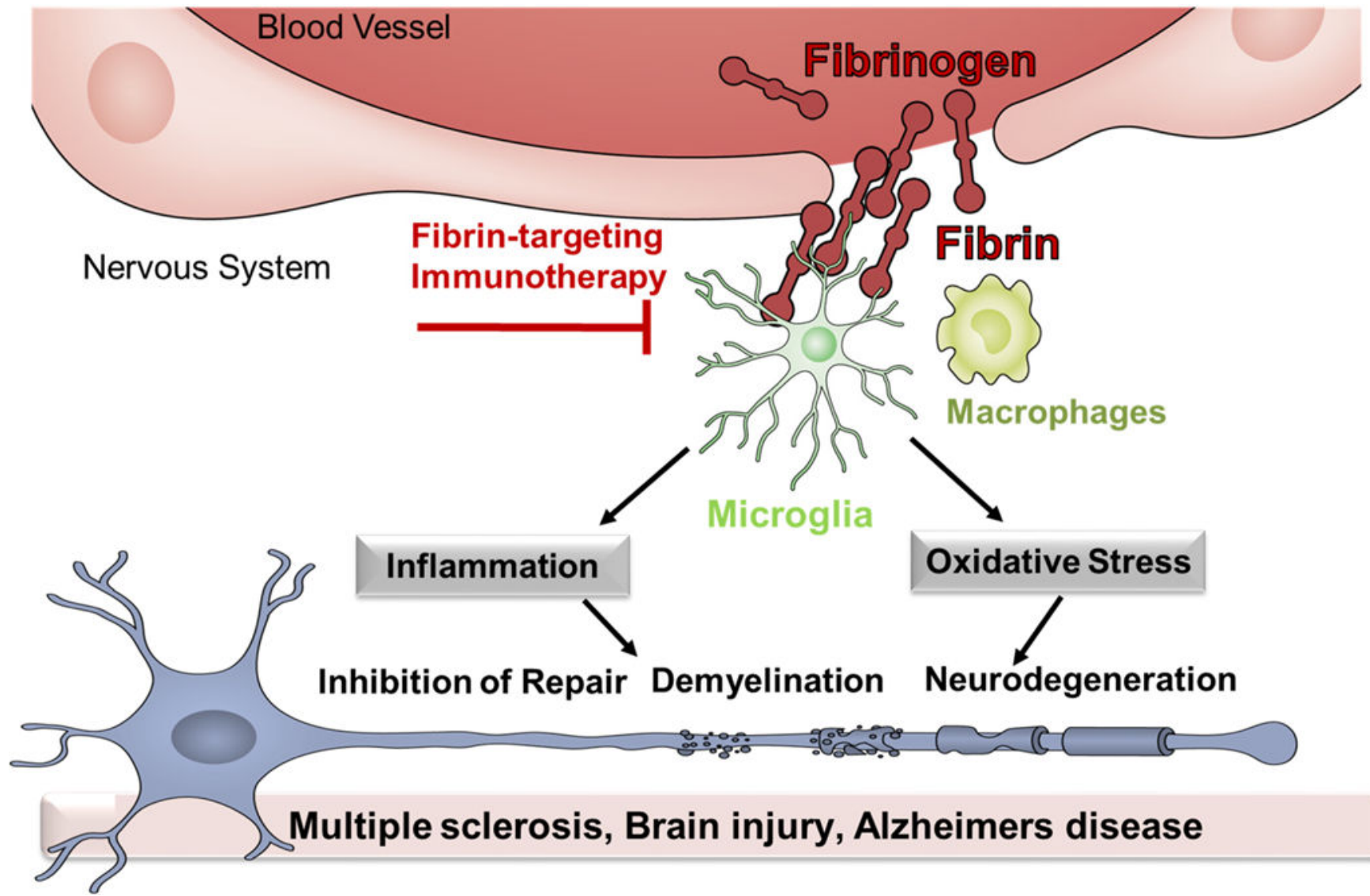

\section{Multiple sclerosis, Brain injury, Alzheimers disease}

Figure 1.

Fibrin mechanisms and functions in neuroinflammation. Upon BBB disruption, fibrinogen leaks in the brain and by activation of the coagulation cascade it is converted to insoluble fibrin deposits. Fibrin activates microglia and promotes recruitment of peripheral immune cells and oxidative injury leading to tissue damage. Inhibition of the interaction of fibrin with its cellular receptors could enable the discovery of selective therapies to block the toxic effects of blood leaks in a wide range of diseases with vascular damage and inflammation. 